\title{
Comparison of Different Magnetorquer Control Laws for QSAT
}

\author{
By Kikuko MiYATA ${ }^{1)}$, Tomohiro NARUMI ${ }^{2)}$, Jozef C. van der HA ${ }^{1)}$ \\ ${ }^{1)}$ Department of Aeronautics and Astronautics, Kyushu University, Fukuoka, Japan \\ ${ }^{2)}$ Department of Aeronautics and Astronautics, Tohoku University, Sendai, Japan
}

(Received April 25th, 2008)

\begin{abstract}
This paper explains the attitude control method for a $50 \mathrm{~kg}$ class satellite QSAT. It uses three-axis magnetorquers for control and a gravity-gradient extension boom for enhancement of the attitude stabilization. We divide the mission period into three main attitude-control phases. The first phase refers to the de-tumbling which ends when one particular satellite axis is roughly along the local Earth-pointing direction. In the second phase, we extend the boom. The third phase refers to the normal mission mode, when accurate attitude control will be achieved by means of the magnetorquers. We construct a number of normal-mode control algorithms and evaluate their performances by simulations. We calculate the required control torque by the PD (Proportional-Derivative) control method. The differences of the methods appear in the calculations of the required magnetic moments. In this paper, we simulate the following methods; 1) simplified linearized dynamics equation method, 2) the LQR (Linear Quadratic Regulator) method, and 3) the cross product method which utilizes only data from the magnetometer and the rate gyros. From the simulations, we conclude that "simplified linearized dynamics equation method" is the most efficient method for QSAT.
\end{abstract}

Key Words: Small Satellite, Attitude Control, Magnetorquer

\section{Nomenclature}

$\begin{array}{cl}T & \text { :torque } \\ K, k & : \text { control gains } \\ \phi, \theta, \psi & \text { :attitude angles } \\ \omega & \text { :rotation (in body frame) } \\ \omega_{\mathrm{o}} & \text { :orbital rate } \\ I & : \text { inertia moment } \\ \tau & \text { :time constant } \\ \omega_{\mathrm{n}} & \text { :undamped natural frequency } \\ \zeta & \text { :damping ratio } \\ t & \text { :control period } \\ \boldsymbol{c} & \text { :cross product vector } \\ \boldsymbol{B} & \text { :geomagnetic vector } \\ \boldsymbol{M} & \text { :magnetic moment vector }\end{array}$

Subscripts

$\begin{array}{cl}c & : \text { control } \\ d & \text { :disturbance } \\ b & \text { :body frame } \\ R & \text { :requirements } \\ c a n & \text { :actually can be generated } \\ x, y, z & : \text { axis } \\ m & \text { :measurement } \\ r & \text { :reference }\end{array}$

\section{Introduction}

\subsection{QSAT}

In the northern region of Kyushu Island, we are developing a $50 \mathrm{~kg}$ class satellite, which is named QSAT. Kyushu University plays a central role in this development.

This satellite has two primary mission objectives. One is the investigation of the plasma physics in the Earth's aurora zone in order to better understand spacecraft charging. The other is performing a comparison of FAC (Field-Aligned Current) observed in orbit with ground-based observations. ${ }^{1)}$

This satellite is planned to be launched by the H-IIA rocket as a piggy-back satellite. The orbit depends on the main satellite. It is assumed to be circular, sun-synchronous orbit and its altitude is in the range $600-800[\mathrm{~km}]$.

\subsection{Attitude requirements}

In order to achieve the QSAT mission objectives, this satellite's attitude needs to be controlled within a few degrees relative to the local orbital frame. Rapid changes in the attitude should be avoided, and the absolute value of the rotation rate around the yaw axis (Earth-pointing axis) must be less than 45 degrees over one orbital period.

\subsection{Attitude units}

QSAT uses the 3-axis magnetorquers for control and a gravity-gradient extension boom for enhancement of attitude stabilization. The gravity-gradient extension boom increases its inertia moments along the roll and pitch axes. The yaw axis pointing attitude stability improves because of the gravity-gradient torque. The magnetorquers can generate a magnetic moment by the interaction with the geomagnetic field in response to a control current. This satellite has three magnetorquers and they are oriented along the axes of the Body-Centered Reference Frame (BCRF). Our satellite has limited electrical power and this drives the design. Magnetorquers are suitable for our satellite because they can generate control torques using less electric power than other actuators. Of course, there are other advantages as well, for instance, low cost, simplicity, and easy to handle.

The magnetorquers have already been used as the main actuators for many satellites, such as Ørsted (1999) ${ }^{2)}$, WEOS $(2002)^{3)}$. 
We present practical and effective three-axis control laws that are suitable for low cost small satellites.

The attitude-pointing information comes from two sets of three-axis sun sensors, two sets of three-axis magnetometers, and two sets of three-axis gyro sensors. The attitude is controlled using the attitude determination results based on these sensors measurements. ${ }^{4)}$

\subsection{Mission phases}

We divide the mission period into 3 phases roughly corresponding to the attitude conditions. The first phase refers to the de-tumbling phase. At the end of this phase, the axis along which the boom will be extended is controlled roughly along the local Earth-pointing direction. In the second phase, we extend the boom for passive attitude stabilization. The third phase refers to the normal mission mode, when accurate Earth-pointing attitude will be achieved by means of magnetorquers.

\section{Geometry Definitions}

As shown in Fig. 1, the transformation from the inertial frame with axes $(\boldsymbol{I}, \boldsymbol{J}, \boldsymbol{K})$ to the orbital frame with axes $\left(\boldsymbol{x}_{0}, \boldsymbol{y}_{0}, \boldsymbol{z}_{0}\right)$ is performed by means of the 3-1-3 $(\Omega, i, u)$ Euler rotation sequence. In this figure, $u$ means the argument of latitude and is defined by $u=\omega+f ; \omega$ is the argument of perigee and $f$ is the true anomaly. The inclination is $i$ and $\Omega$ means the right ascension of the ascending node.

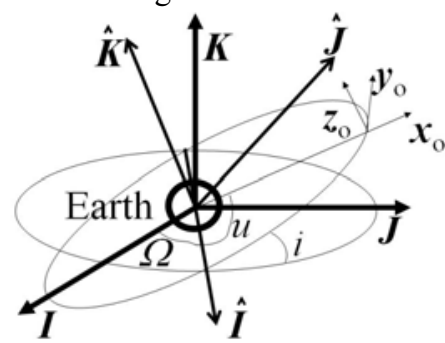

Fig. 1. Inertia frame and orbital frame

The orbital frame $\left(\boldsymbol{x}_{0}, \boldsymbol{y}_{0}, \boldsymbol{z}_{0}\right)$ and the Body-Centered Reference Frame (BCRF) $\left(\boldsymbol{x}_{\mathrm{b}}, \boldsymbol{y}_{\mathrm{b}}, \boldsymbol{z}_{\mathrm{b}}\right)$ are defined in Figs. 2-4.

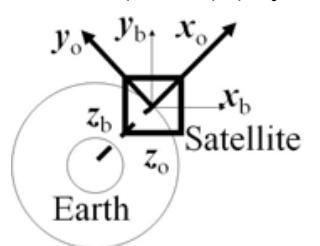

Fig. 2. Orbital frame

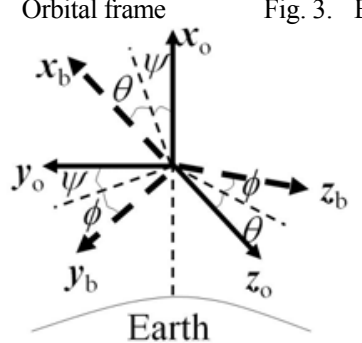

Fig. 4. Body frame to orbital frame

The sequence of transformation from orbital to the BCRF is $3-2-1(\psi, \theta, \phi)$. As shown in Fig. $4, \psi$ is the rotation along the $z$-axis, $\theta$ is the rotation along the $y$-axis, and $\phi$ is the rotation along the $x$-axis.

\section{Control Concept}

In the de-tumbling phase, we use the B-dot control method. The details of this method and the detailed results have been given in Ref. 5. By this method, the magnetorquers generate the magnetic moments that have the opposite signs of the time variations in the measured geomagnetic field in the spacecraft body frame. The generated magnetic moments are the specified maximum values ( + or $\left.-1.0\left[\mathrm{Am}^{2}\right]\right)$. As a result, the satellite can reduce its body rates and the rates can achieve the rough Earth-pointing values. Fig. 5 shows the simulation result under worst-case assumptions, for instance, the initial angular rates are all taken as $30 \mathrm{deg} / \mathrm{sec}$ after the launcher ejection. Our satellite can de-tumble within 8 hours in this case.

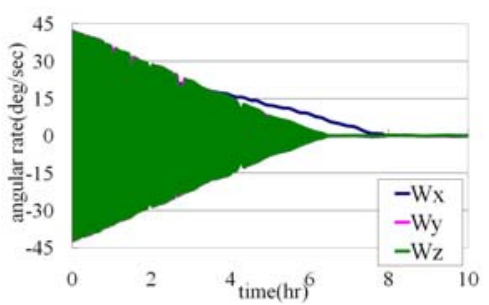

Fig. 5. Results of de-tumbling angular rates

In this paper, we focus on the control method of the normal-mode phase. We construct a number of control algorithms and simulate each method. Finally, we compare the results and discuss their merits and demerits.

\section{Control Laws}

The core part of each method is identical. We calculate the required control torque by the PD (Proportional-Derivative) control method. This control concept calculates the control torques in proportion to the errors in the angles and the angular rates (relative to the ideal Earth-pointing direction) and reduces these errors. The proportionality coefficients between the errors and the required control torques are called the control gains.

We can calculate the gains by various methods. In this paper, we simulate the following methods; 1) simplified linearized dynamics equation method, 2) LQR (Linear Quadratic Regulator) method, and 3) cross product method.

\subsection{Simplified linearized dynamics equation}

The required control torques are calculated as follows:

$$
\begin{aligned}
& \boldsymbol{T}_{c}=\left[\begin{array}{c}
T_{c_{x}} \\
T_{c_{y}} \\
T_{c_{z}}
\end{array}\right]= {\left[\begin{array}{ccc}
K_{x} & 0 & 0 \\
0 & K_{y} & 0 \\
0 & 0 & K_{z}
\end{array}\right]\left[\begin{array}{c}
\phi \\
\theta \\
\psi
\end{array}\right]+\left[\begin{array}{ccc}
k_{x} & 0 & 0 \\
0 & k_{y} & 0 \\
0 & 0 & k_{z}
\end{array}\right]\left[\begin{array}{c}
\dot{\phi} \\
\dot{\theta} \\
\dot{\psi}
\end{array}\right] } \\
& \text { with control gains: } K_{x}, K_{y}, K_{z}, k_{x}, k_{y}, k_{z}
\end{aligned}
$$

We consider only the gravity-gradient disturbance and assume short time intervals and small attitude angles. Also we neglect the coupling terms of $(\dot{\phi}, \dot{\theta}, \dot{\psi})$. The Euler dynamical equations can now be expressed: ${ }^{6)}$

$$
\begin{aligned}
& I_{x} \ddot{\phi}+\omega_{o}^{2}\left(I_{z}-I_{y}\right) \phi=T_{x} \\
& I_{y} \ddot{\theta}-4 \omega_{o}^{2}\left(I_{x}-I_{z}\right) \theta=T_{y} \\
& I_{z} \ddot{\psi}+3 \omega_{o}^{2}\left(I_{y}-I_{x}\right) \psi=T_{z}
\end{aligned}
$$


Here, $\boldsymbol{T}=\left[\boldsymbol{T}_{\mathrm{c}}+\boldsymbol{T}_{\mathrm{d}}\right] . \boldsymbol{T}_{\mathrm{d}}$ is the disturbance torque and $\boldsymbol{T}_{\mathrm{c}}$ can be expressed by Eq. (1). By these relationships, Eqs. (2) can be simplified as:

$$
I_{i} \ddot{\theta}_{i}+K_{i} \dot{\theta}_{i}+c_{i} \theta_{i}=T_{d_{i}}(i=x, y, z)
$$

Here, $\theta_{\mathrm{x}}=\phi, \theta_{\mathrm{y}}=\theta, \theta_{\mathrm{z}}=\psi$.

The extra parameters in Eq. (3) are defined as follows: $c_{x}=\left(I_{z}-I_{y}\right) \omega_{o}^{2}+K_{x}, c_{y}=4\left(I_{z}-I_{x}\right) \omega_{o}^{2}+K_{y}, c_{z}=3\left(I_{y}-I_{x}\right) \omega_{o}^{2}+K_{z}$

In this case, the transfer function of Eq. (3) is:

$$
\begin{gathered}
\frac{\Theta_{i}(s)}{T_{d_{i}}(s)}=\frac{1}{I_{i} s^{2}+K_{i} s+c_{i}}=\frac{\omega_{n_{i}}^{2}}{c_{i}\left(s^{2}+2 \zeta_{i} \omega_{n_{i}} s+\omega_{n_{i}}^{2}\right)} \\
\text { Here, } \tau_{i} \equiv K_{i} / 2 c_{i}, \omega_{n_{i}} \equiv \sqrt{c_{i} / I_{i}}, \zeta_{i} \equiv \tau_{i} \omega_{n_{i}}(i=x, y, z)
\end{gathered}
$$

From this result, we can calculate the gains. We consider the critical damping case, which represent the limit of rapid response with no vibration and damping ratios $\zeta_{i}=1(i=x, y, z)$. Moreover, we introduce the control period $t_{i}(i=x, y, z)$, the other parameters can be calculated as follows;

- Undamped natural frequency: $\omega_{n i}=2 \pi / t_{\mathrm{i}}(i=x, y, z)$

- Time constant: $\tau_{i}=\zeta_{i} / \omega_{n i}(i=x, y, z)$

We will determine the best control period $t_{i}(i=x, y, z)$ from the simulation results.

From these discussions, the gains are calculated as following equations:

$$
\begin{aligned}
K_{P_{x}} & =4 \pi^{2} I_{x} / t_{x}^{2}-\left(I_{z}-I_{y}\right) \omega_{o}^{2} \\
K_{P_{y}} & =4 \pi^{2} I_{y} / t_{y}{ }^{2}-4\left(I_{z}-I_{x}\right) \omega_{o}^{2} \\
K_{P_{z}} & =4 \pi^{2} I_{z} / t_{z}{ }^{2}-3\left(I_{y}-I_{x}\right) \omega_{o}^{2} \\
K_{R_{i}} & =16 \pi \zeta_{i} I_{i} / t_{i} \quad(i=x, y, z)
\end{aligned}
$$

\subsection{LQR (Linear Quadratic Regulator) method}

The LQR (Linear Quadratic Regulator) method can easily establish the optimal feedback gain of the linear system. This theory is concerned with operating a dynamic system at minimum cost. The case where the system dynamics are described by a set of linear differential equations and the cost is given by a quadratic function is called the LQ problem. The $\mathrm{LQR}$ is one of the main results in the theory. We introduce the state vector $\mathbf{x}=\left[\begin{array}{llllll}\phi & \theta & \psi & \omega_{\mathrm{x}} & \omega_{\mathrm{y}} & \omega_{\mathrm{z}}-\omega_{0}\end{array}\right]^{\mathrm{T}}$ and the control torque $\mathbf{u}=\left[\begin{array}{llllll}0 & 0 & 0 & T_{\mathrm{x}} & T_{\mathrm{y}} & T_{\mathrm{z}}\end{array}\right]^{\mathrm{T}}$. In addition, we make use of the fact that $\dot{\Omega}$ is very small compared to $\dot{\phi}, \dot{\theta}, \dot{\psi}, \phi, \theta, \psi$ and assume no disturbance cases. The linear state equation can now be expressed in $\dot{\mathbf{x}}=\mathbf{A x}+\mathbf{B u}$ form;

$$
\begin{aligned}
{\left[\begin{array}{c}
\dot{\phi} \\
\dot{\theta} \\
\dot{\psi} \\
\dot{\omega}_{x} \\
\dot{\omega}_{y} \\
\dot{\omega}_{z}
\end{array}\right] } & {\left[\begin{array}{cccccc}
0 & \omega_{o} & 0 & 1 & 0 & 0 \\
-\omega_{o} & 0 & 0 & 0 & 1 & 0 \\
0 & 0 & 0 & 0 & 0 & 1 \\
0 & 0 & 0 & 0 & \frac{I_{y}-I_{z}}{I_{x}} \omega_{o} & 0 \\
0 & 0 & 0 & \frac{I_{z}-I_{x}}{I_{y}} \omega_{o} & 0 & 0 \\
0 & 0 & 0 & 0 & 0 & 0
\end{array}\right]\left[\begin{array}{c}
\phi \\
\theta \\
\psi \\
\omega_{x} \\
\omega_{y} \\
\omega_{z}-\omega_{o}
\end{array}\right] } \\
& +\left[\begin{array}{ccc}
0 & 0 & 0 \\
0 & 0 & 0 \\
0 & 0 & 0 \\
1 / I_{x} & 0 & 0 \\
0 & 1 / I_{y} & 0 \\
0 & 0 & 1 / I_{z}
\end{array}\right]\left[\begin{array}{l}
T_{c_{x}} \\
T_{c_{y}} \\
T_{c_{z}}
\end{array}\right]
\end{aligned}
$$

The cost function is defined as $J=\int_{0}^{\infty}\left(\mathbf{x}^{T} \mathbf{Q x}+\mathbf{u}^{T} \mathbf{R u}\right) d t$ where $\mathbf{Q}$ and $\mathbf{R}$ are weighting matrices. Optimizing the function $\boldsymbol{J}$ means that the energy for the control is minimized and the state vector converges to 0 as fast as possible.
The optimal feedback control gain $\mathbf{K}$ becomes

$$
\mathbf{u}=-\mathbf{K x}=-\mathbf{R}^{-1} \mathbf{B}^{T} \mathbf{P x}
$$

where $\mathbf{P}$ is found by solving the Riccati equation

$$
\mathbf{A}^{T} \mathbf{P}+\mathbf{P A}-\mathbf{P B R}^{-1} \mathbf{B}^{T} \mathbf{P}+\mathbf{Q}=0
$$

The Riccati equation can be solved numerically using MATLAB

$$
\mathbf{P}=\mathbf{Q}+\mathbf{A}^{T}\left(\mathbf{P}-\mathbf{P B}\left(\mathbf{R}+\mathbf{B}^{T} \mathbf{P B}\right)^{-1} \mathbf{B}^{T} \mathbf{P}\right) \mathbf{A}
$$

\subsection{Cross product method}

This method utilizes only magnetometer and rate gyros data The control performance depends on the measured geomagnetic vector relative to the reference geomagnetic vector. This method does not guarantee that the attitude angles converge to zero because the attitude error along the geomagnetic field vector cannot be controlled. However, the attitude angles are expected to approach zero over time because the geomagnetic field vector changes continuously in the body frame even when the attitude is stable.

This method uses the cross product vector $c$ between the measured geomagnetic vector $\boldsymbol{b}_{\mathrm{m}}$ in the body frame and the geomagnetic vector $\boldsymbol{b}_{\mathrm{r}}$, referenced by the International Geomagnetic Reference Field (IGRF) model ${ }^{7}$, as the Euler axis direction. When the attitude error angles are small, the direction of $c$ becomes almost the same as the Euler axis.

The $c$ vector can be described as

$$
\boldsymbol{c}=\left(\boldsymbol{b}_{m} /\left|\boldsymbol{b}_{m}\right|\right) \times\left(\boldsymbol{b}_{r} /\left|\boldsymbol{b}_{r}\right|\right)
$$

In this case, if we introduce the measured rates $\Delta \boldsymbol{\omega}=\left[\omega_{\mathrm{x}} \omega_{\mathrm{y}}\right.$ $\left.\omega_{\mathrm{z}}-\omega_{\mathrm{o}}\right]^{\mathrm{T}}$, we can calculate the required control torque vector $\boldsymbol{T}_{\mathrm{r}}$ by

$$
\boldsymbol{T}_{R}=\boldsymbol{K}_{p} \boldsymbol{c}+\boldsymbol{K}_{r} \Delta \boldsymbol{\omega}
$$

The gains can be calculated in the same way as in section 4.1, because the characteristics are similar.

\section{Conversion of Required Torque to Magnetic Moment}

From the required control torque, we can calculate the magnetic moment needed to perform the control. There are a number of ways to convert the required torque into the magnetic moment that can be generated. However, we cannot generate the required torque in general because the torque acts always normal to the magnetic field direction.

Here, we calculate the magnetic moments by the following procedures. The required control torque $\boldsymbol{T}_{\mathrm{R}}$ can be calculated by the control law and we can measure the magnetic-field vector $(\boldsymbol{B})$. Here, we introduce the control torque, which we actually can generate by the magnetorquers, as $\boldsymbol{T}_{\text {can }}$ with $\boldsymbol{B} \cdot \boldsymbol{T}_{\text {can }}$ $=0, \boldsymbol{M} \cdot \boldsymbol{T}_{\text {can }}=0$. We know both the $\boldsymbol{B}$ and $\boldsymbol{T}_{\mathrm{R}}$ vectors and we will calculate $\boldsymbol{T}_{\text {can }}$ which is in the plane defined by the vectors $\boldsymbol{B}$ and $\boldsymbol{T}_{\mathrm{R}}$. We define $\beta$ as the angle between $\boldsymbol{B}$ and $\boldsymbol{T}_{\mathrm{R}}$ (with $0 \leq \beta \leq \pi)$, see Fig. 6 .

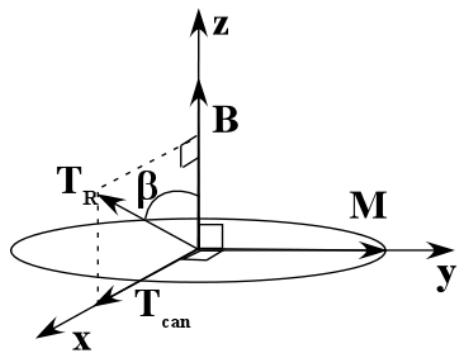

Fig. 6. Required control torque $\boldsymbol{T}_{\mathrm{R}}$ and magnetic moment $\boldsymbol{M}$ 
As seen in Fig. 6, we use the new Cartesian coordinates with the $x$ axis along the $\boldsymbol{T}_{\text {can }}$ vector, the $y$ axis along the magnetic moment vector $\boldsymbol{M}$ and the $z$ axis along the $\boldsymbol{B}$ vector.

The respective unit vectors can be described as:

$$
\left(\boldsymbol{e}_{x}, \boldsymbol{e}_{y}, \boldsymbol{e}_{z}\right)=\left(\boldsymbol{T}_{c a n} / T_{c a n}, \boldsymbol{M} / M, \boldsymbol{B} / B\right)
$$

In this case,

$$
\boldsymbol{T}_{R}=\left(T_{R} \sin \beta\right) \boldsymbol{e}_{x}+\left(T_{R} \cos \beta\right) \boldsymbol{e}_{z}
$$

Thus, the $\boldsymbol{T}_{\text {can }}$ vector is:

$$
\boldsymbol{T}_{\text {can }}=\left(T_{R} \sin \beta\right) \boldsymbol{e}_{x}=\boldsymbol{T}_{R}-\left(T_{R} \cos \beta\right) \boldsymbol{e}_{z}=\boldsymbol{T}_{R}-\left(T_{R} \cos \beta\right) \boldsymbol{B} / B
$$

From the magnetorquer equation, the torque that actually can be generated $\boldsymbol{T}_{\text {can }}$ can also be described in the following form:

$$
\boldsymbol{T}_{c a n}=\boldsymbol{M} \times \boldsymbol{B}
$$

When we take the cross product of $\boldsymbol{B}$ on the left side on both sides of the above expression, we find:

$$
\boldsymbol{B} \times \boldsymbol{T}_{\text {can }}=\boldsymbol{B} \times(\boldsymbol{M} \times \boldsymbol{B})=(\boldsymbol{B} \bullet \boldsymbol{B}) \boldsymbol{M}-(\boldsymbol{M} \bullet \boldsymbol{B}) \boldsymbol{B}=B^{2} \boldsymbol{M}(17)
$$

Because we assume that the $\boldsymbol{M}$ is normal to the $\boldsymbol{B}$-vector, we have $\boldsymbol{M} \cdot \boldsymbol{B}=0$ in this case. From the above result, we get:

$$
\boldsymbol{M}=\frac{\boldsymbol{B} \times \boldsymbol{T}_{c a n}}{B^{2}}
$$

From these discussions we can express the required magnetic moment $\boldsymbol{M}$ in terms of the known $\boldsymbol{B}$ and $\boldsymbol{T}_{\mathrm{r}}$ vectors (see Fig. 6):

$$
\boldsymbol{M}=\frac{\boldsymbol{B} \times \boldsymbol{T}_{c a n}}{B^{2}}=\frac{\boldsymbol{B}}{B^{2}} \times\left\{\boldsymbol{T}_{R}-\frac{\left(T_{R} \cos \beta\right) \boldsymbol{B}}{B}\right\}=\frac{\boldsymbol{B} \times \boldsymbol{T}_{R}}{B^{2}}
$$

\section{Simulation Environments}

\subsection{Conditions}

We assume a circular, sun-synchronous orbit.

\begin{tabular}{|c|c|c|c|c|c|}
\multicolumn{7}{|c}{ Table 1. Orbital elements. } \\
\hline$i(\mathrm{deg})$ & $\Omega(\mathrm{deg})$ & $\mathrm{e}$ & $\omega(\mathrm{deg})$ & $M(\mathrm{deg})$ & $\omega_{\mathrm{o}}(\mathrm{deg} / \mathrm{sec})$ \\
\hline 98.06 & 105 & 0.0005 & 0.001 & 180 & 0.061186 \\
\hline
\end{tabular}

$i$ : inclination, $\Omega$. right ascension of the ascending node,

e: eccentricity, $\omega$ : argument of perigee,

$M$ : mean anomaly (at start), $\omega_{0}$ : orbital rate

QSAT is assumed to be in a Sun-synchronized orbit with $\dot{\Omega}=0.986$ [deg/day].

Initial angles and rates are set as 0.0 [deg or $\mathrm{deg} / \mathrm{sec}]$. This is the ideal condition at the end of the de-tumbling phase. In addition, we set duty ratio as 0.5 .

The target angles are $0.0[\mathrm{deg}]$ and the target rates are 0.0 [deg/sec] except $\omega_{\mathrm{z}}$. The $\omega_{\mathrm{z}}$ target value is $\omega_{\mathrm{o}}[\mathrm{deg} / \mathrm{sec}]$.

\subsection{Disturbance torque}

We consider worst values of the following disturbances.

$>$ Gravity-Gradient Torque

$>$ Solar Radiation Pressure Torque

$>$ Atmospheric Drag Torque

Remnant Magnetism Torque

The gravity-gradient torque is calculated as: ${ }^{8)}$

$$
\mathbf{T}_{g g}=\frac{3 \mu_{E}}{R^{3}}\left[\begin{array}{c}
m n\left(I_{z}-I_{y}\right)+n l I_{x y}-\operatorname{lm} I_{x z}+\left(n^{2}-m^{2}\right) I_{y z} \\
n l\left(I_{x}-I_{z}\right)+\operatorname{lm} I_{x y}-m n I_{x y}+\left(l^{2}-n^{2}\right) I_{x z} \\
l m\left(I_{y}-I_{x}\right)+m n I_{x y}-n l I_{y z}+\left(m^{2}-l^{2}\right) I_{x y}
\end{array}\right] \text { (20) }
$$

Here, $\boldsymbol{R}=R(l, m, n)^{\mathrm{T}}$ is the orbital radius vector in the Body Centered Reference Frame (BCRF). Its origin is the center of the Earth and points to the center of mass of the satellite.

The solar radiation pressure torque can be calculated as:

$$
\begin{aligned}
& \mathbf{T}_{s r p}=\left(\mathbf{c}_{s r p}-\mathbf{c}_{m}\right) \times \mathbf{F}_{s r p} \\
& \mathbf{F}_{s r p}=\frac{F_{s}}{c} A_{s}(1+q) \mathbf{r}_{s u n}
\end{aligned}
$$

Here, $\mathbf{c}_{s r p}$ is the center of solar radiation pressure in the BCRF and $\mathbf{c}_{m}$ is the center of mass. We take $5[\mathrm{~cm}]$ as the distance between $\mathbf{c}_{s r p}$ and $\mathbf{c}_{m}$. This is the worst-case offset. $F_{s}$ is the solar constant with value $1367.0\left[\mathrm{~W} / \mathrm{m}^{2}\right], A_{s}$ is the effective area, $q$ is the reflectivity and we choose the worst value $1.0, c$ is the velocity of light, and $\mathbf{r}_{s u n}$ is the unit sun vector as seen in the BCRF frame. The vector of the sun is calculated using the VSOP87 (Variations Séculaires des Orbites Planétaires) method. $^{9)}$

The atmospheric drag torque is calculated from:

$$
\begin{aligned}
& \mathbf{T}_{a}=\left(\mathbf{c}_{a}-\mathbf{c}_{m}\right) \times \mathbf{F}_{a} \\
& \mathbf{F}_{a}=\frac{1}{2} \rho C_{d} A V \mathbf{V}
\end{aligned}
$$

Here $\mathbf{c}_{a}$ is the center of aerodynamic force and the distance between $\mathbf{c}_{a}$ and $\mathbf{c}_{m}$ is also taken $5[\mathrm{~cm}] . C_{d}$ is the drag coefficient, $\rho$ is the atmospheric density, and $V$ is the orbital velocity. The atmospheric density is calculated by NRLMSISE-00. (MSISE: Mass Spectrometer, Incoherent Scatter Radar Extended Model)

The remnant magnetic torque can be calculated as:

$$
\boldsymbol{T}_{\text {mag }}=\boldsymbol{M}_{\boldsymbol{d}} \times \boldsymbol{B}
$$

$\boldsymbol{M}_{\mathrm{d}}$ is the effective remnant magnetism of the satellite and the $\boldsymbol{B}$ is the geomagnetic vector and calculated by IGRF method.

\section{Simulation Results}

\subsection{No disturbance case}

Result 1 Simplified equation method $(\mathrm{t}=3200[\mathrm{sec}])$

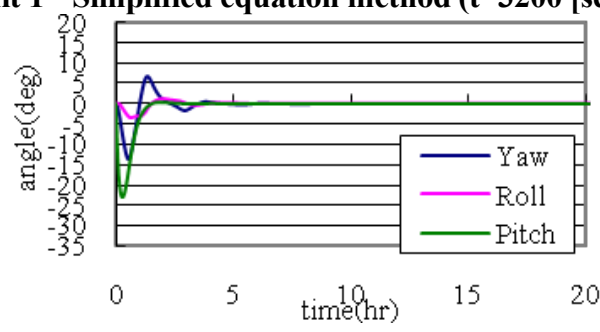

Fig. 7. Angles results of simplified equation method

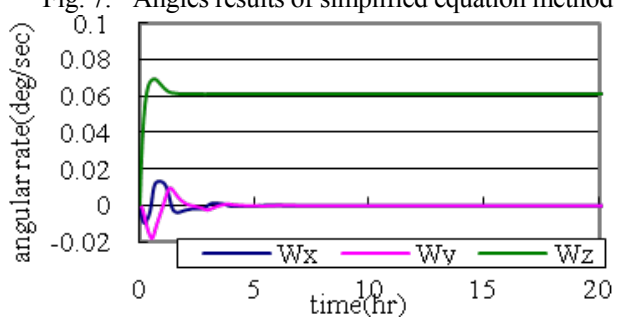

Fig. 8. Rates results of simplified equation method

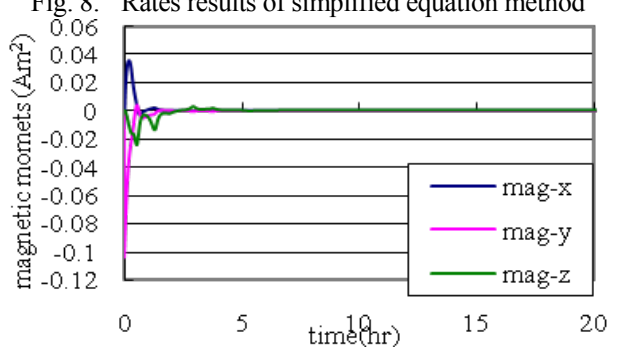

Fig. 9. Magnetic moments results of simplified equation method

As seen in these results, we can achieve the target values within about 6 hours by this method. 


\section{Result 2 LQR method}

We use the following weighting matrices in this case. The $\mathbf{Q}$ matrix has only diagonal terms and these terms are given by these values.

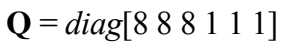

The $\mathbf{R}$ matrix is the $6 \times 6$ matrix and can be expressed by the Kronecker delta.

$$
\mathbf{R}=\left[\delta_{i j}\right]=\left\{\begin{array}{c}
1.0 \times 10^{12}(i=j) \\
0(i \neq j)
\end{array} \quad i, j=1, \ldots, 6\right.
$$

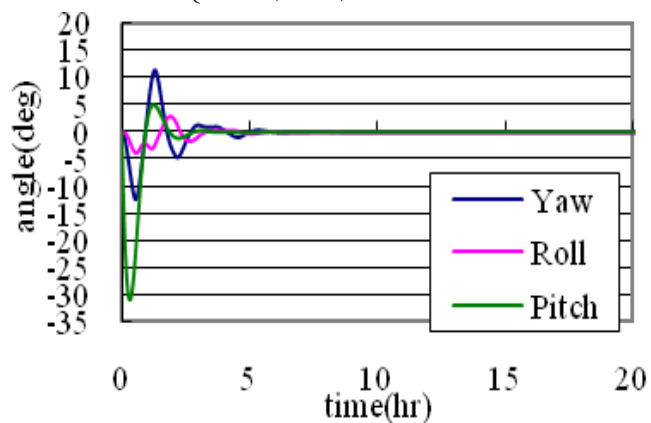

Fig. 10. Angles results of LQR method

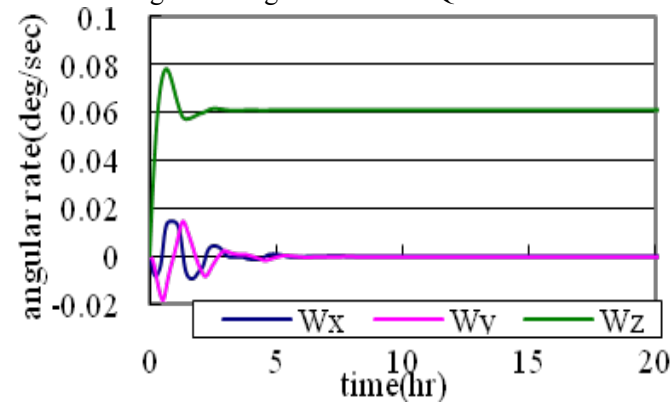

Fig. 11. Rates results of LQR method

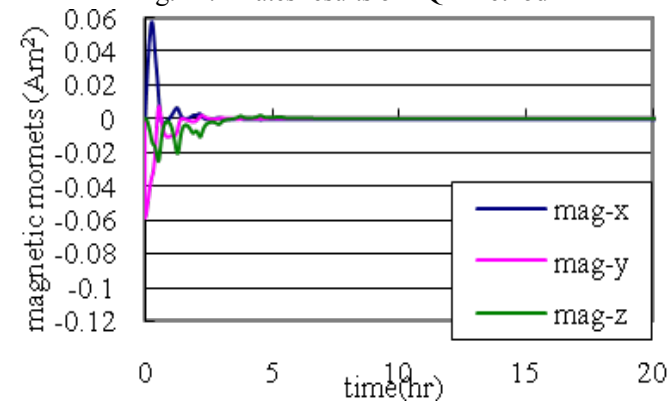

Fig. 12. Magnetic moments results of $L Q R$ method

By the LQR method, we can achieve the target values within about 7 hours. The rough control trends are almost the same as the simplified equation case. However, this method adds too much control torque for pitch at the beginning of control.

Result 3 Cross product method $(\mathrm{t}=3000[\mathrm{sec}])$

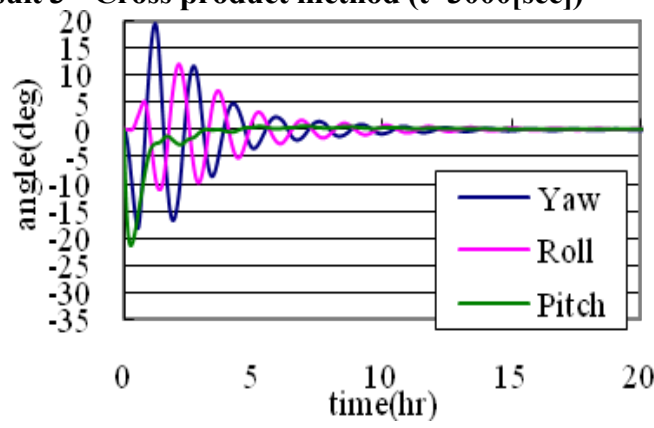

Fig. 13. Angles results of cross product method

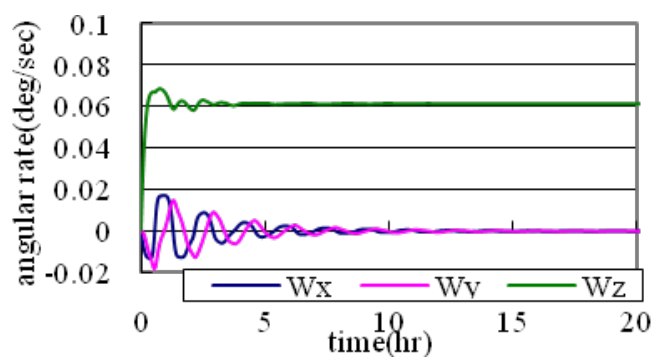

Fig. 14. Rates results of cross product method

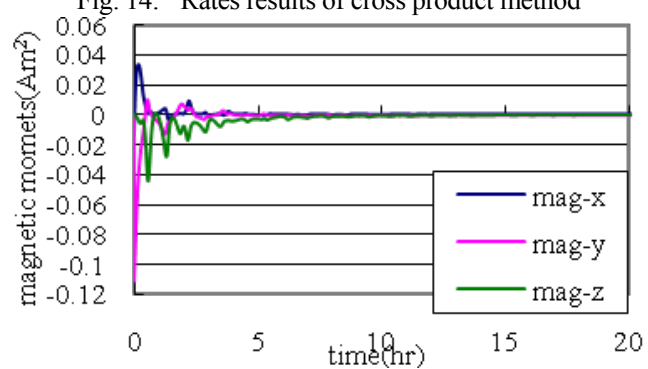

Fig. 15. Magnetic moments results of cross product method

We can achieve the target values within 16 hours by this method. The results show considerable vibrations. The control performances seem to be worse than the other methods.

\subsection{Disturbances case}

In this section, we consider all disturbances. We know the remnant magnetism roughly from ground-based measurement. Therefore, we can negate the remnant effect by generating the opposite magnetic moments of the remnant magnetism. In these cases, we set the remnant magnetism as $(-0.1,-0.1,-0.1)$ $\left[\mathrm{Am}^{2}\right]$.

Result 1 Simplified equation method $(\mathrm{t}=\mathbf{3 2 0 0}[\mathrm{sec}])$

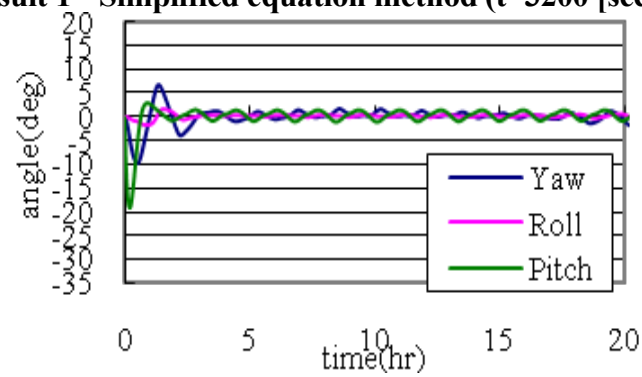

Fig. 16. Angles results of simplified equation method

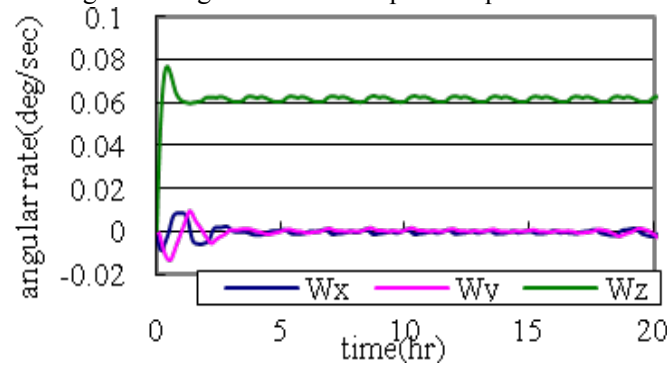

Fig. 17. Rates results of simplified equation method

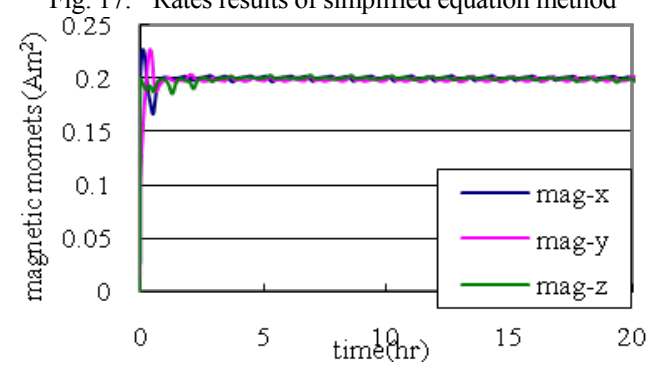

Fig. 18. Magnetic moments results of simplified equation method 
In this case, the rough trends are the same as the no disturbance case. However, the vibration terms caused by the disturbances appear in all results. These vibrations lead to angular errors of about -1.0 to 1.5 [deg]. The largest error appears in the pitch axis.

\section{Result 2 LQR method}

We use the same weighting matrices as the no disturbance case.

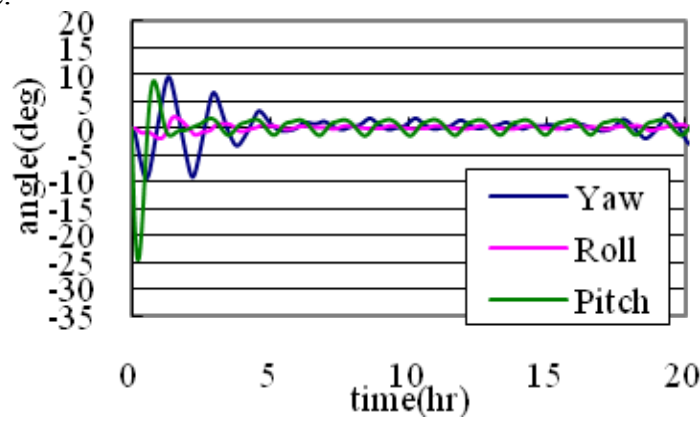

Fig. 19. Angles results of LQR method

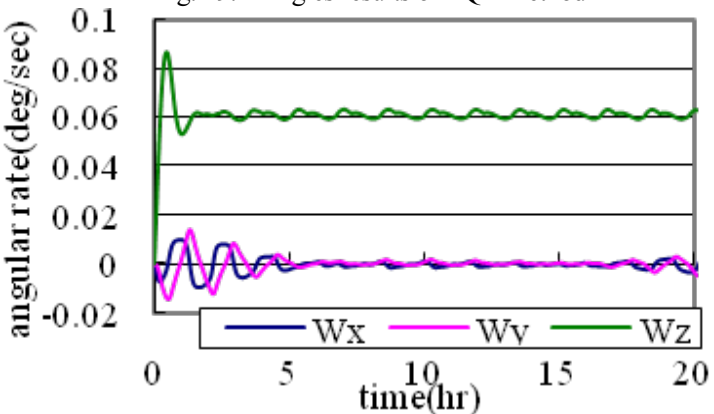

Fig. 20. Rates results of LQR method

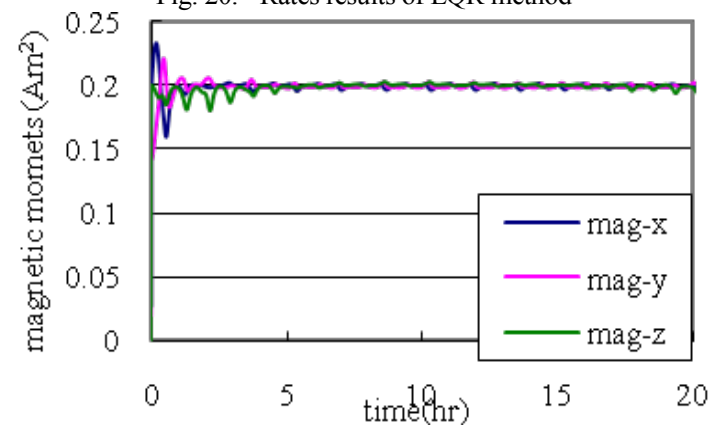

Fig. 21. Magnetic moments results of LQR method

By the LQR method, we can achieve the rough target values within about 8 hours. From these results, we can see the larger remnant errors than the simplified equation case.

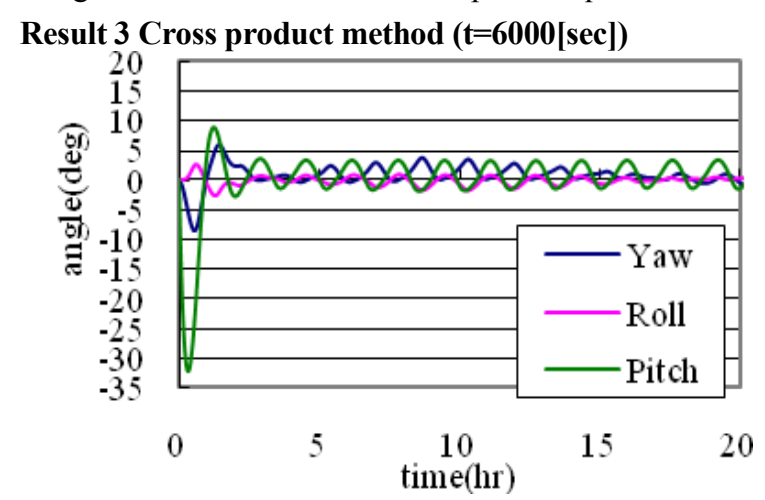

Fig. 22. Angles results of cross product method

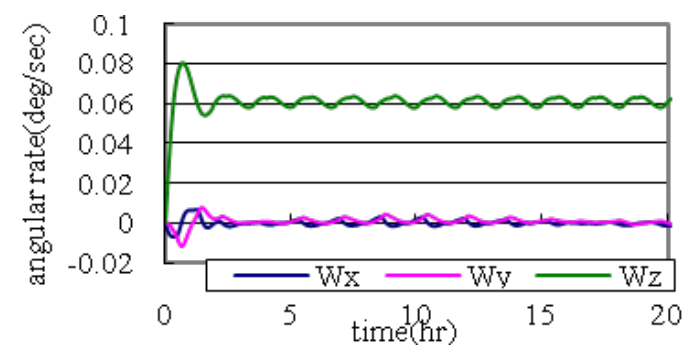

Fig. 23. Rates results of cross product method

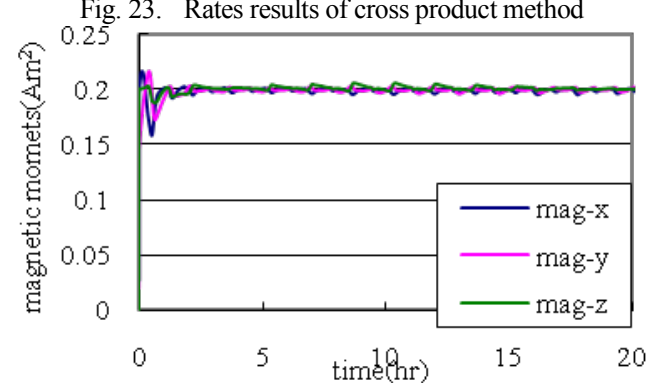

Fig. 24. Magnetic moments results of cross product method

We can achieve the target values within about 15 hours in this method. However, the best control period change becomes twice as large as in the no disturbance case.

\section{Conclusion}

From the simulation results, we find that the simplified equation case gives the best results. The main reason is that this method has a good tolerance for disturbances. In addition, the required control time by this method is the shortest.

\section{References}

1) Yoshihiro Tsuruda, Toshiya Hanada, Jozef van der Ha: QSAT: A Low-Cost Design for 50kg Class Piggyback Satellite, Paper presented at the 26th International Symposium on Space Technology and Science, 2008-f-20, Hamamatsu, Japan, 2-6 June 2008.

2) T. Bak, M. Blanke, R. Wisniewski: Flight results and lessons learned from the Ørsted attitude control system, 4th ESA International Conference on Spacecraft Guidance, Navigation and Control, 1999.

3) Shigeru Hosokawa, Tomonao Hayashi, Susumu Takezawa, Kazuhiko Haneji: Effective Attitude Control System for Small Satellite, T. SICE, Vol.42, No.2, February 2006, pp. 123-128. (in Japanese)

4) Yuya Mimasu, Jozef van der Ha: Attitude Determination Concept for QSAT, Paper presented at the 26th International Symposium on Space Technology and Science, 2008-d-13, Hamamatsu, Japan, 2-6 June 2008.

5) Kikuko Miyata, Yuya Mimasu, Tomohiro Narumi, Hiroshi Hirayama, Jozef C. van der Ha: Attitude Control by Magnetic Torquer for Small Satellite, Proceedings of 2007 JSASS-KSAS Joint International Symposium on Aerospace Engineering, October 11, 2007, pp.256-259.

6) Vladimir A. Chobotov: Spacecraft Attitude Dynamics and Control (Orbit, a Foundation Series), Krieger Publishing Company, October 1991, Original Edition.

7) Nakatsuka, T.: Calculation of the International Geomagnetic Reference Field (4), GSJ Open File Report No. 423, 2005.

8) van der Ha, J.: Model of Gravity Force and Gravity Gradient Torque, FLK-SIM-TN-ZAR-004 Issue 1, ZARM, University of Bremen, Germany, April 2006

9) Meeus, J.: Astronomical Algorithms Second Edition, Willmann-Bell, US, 2005. 